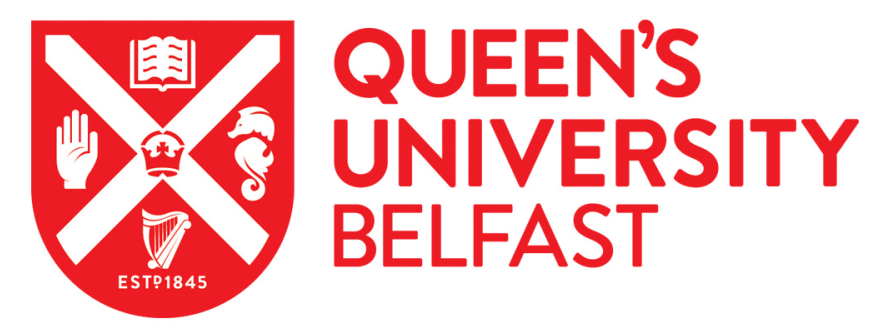

\title{
Back to the future: routine morphological assessment of the tumour microenvironment is prognostic in stage II/III colon cancer in a large population-based study
}

Hynes, S. O., Coleman, H. G., Kelly, P. J., Irwin, S., O'Neill, R. F., Gray, R. T., McGready, C., Dunne, P. D., McQuaid, S., James, J. A., Salto-Tellez, M., \& Loughrey, M. B. (2017). Back to the future: routine morphological assessment of the tumour microenvironment is prognostic in stage II/III colon cancer in a large population-based study. Histopathology. https://doi.org/10.1111/his.13181

Published in:

Histopathology

Document Version:

Peer reviewed version

Queen's University Belfast - Research Portal:

Link to publication record in Queen's University Belfast Research Portal

\section{Publisher rights}

Copyright 2017 Histopathology

This is the peer reviewed version of the following article, which has been published in final form at

http://onlinelibrary.wiley.com/wol1/doi/10.1111/his.13181/abstract. This article may be used for non-commercial purposes in accordance with Wiley Terms and Conditions for Self-Archiving

\section{General rights}

Copyright for the publications made accessible via the Queen's University Belfast Research Portal is retained by the author(s) and / or other copyright owners and it is a condition of accessing these publications that users recognise and abide by the legal requirements associated with these rights.

Take down policy

The Research Portal is Queen's institutional repository that provides access to Queen's research output. Every effort has been made to ensure that content in the Research Portal does not infringe any person's rights, or applicable UK laws. If you discover content in the

Research Portal that you believe breaches copyright or violates any law, please contact openaccess@qub.ac.uk. 
Back to the future: routine morphological assessment of the tumour microenvironment is prognostic in stage II/III colon cancer in a large population-based study

\section{Running Title: Tumour microenvironment and colon cancer}

Seán O Hynes ${ }^{1 *}$, Helen G Coleman ${ }^{2 *}$, Paul J Kelly ${ }^{1,3}$, Steven Irwin $^{3}$, Roisin F O’ Neill ${ }^{2}$, Ronan T Gray ${ }^{2}$, Claire McGready ${ }^{1}$, Philip D. Dunne ${ }^{1}$, Stephen McQuaid ${ }^{1,3,4}$, Jacqueline A James $^{1,3,4}$, Manuel Salto-Tellez ${ }^{1,3}$, Maurice B Loughrey ${ }^{1,3}$

${ }^{1}$ Northern Ireland Molecular Pathology Laboratory, Centre for Cancer Research and Cell Biology, Queen’s University Belfast, Belfast, Northern Ireland, UK

${ }^{2}$ Centre for Public Health, Queen’s University Belfast, Belfast, Northern Ireland, UK

${ }^{3}$ Department of Tissue Pathology, Royal Victoria Hospital, Belfast Health and Social Care Trust, Belfast, Northern Ireland, UK

4 Northern Ireland Biobank, Centre for Cancer Research and Cell Biology, Queen's University Belfast, Northern Ireland.

*These authors have contributed equally to this work and should be considered joint first authors

Corresponding author: Maurice B Loughrey, maurice.loughrey@belfasttrust.hscni.net Department of Histopathology, Institute of Pathology, Royal Victoria Hospital, Grosvenor Road, Belfast BT12 6BA. Phone: +44 2890633224

Authors' Disclosures or Potential Conflicts of Interest: No authors declared any potential conflicts of interest.

Word count (Main text, excluding abstract and references): 4094 


\begin{abstract}
Both morphological and molecular approaches have highlighted the biological and prognostic importance of the tumour microenvironment in colorectal cancer (CRC). Despite this, microscopic assessment of tumour microenvironment has not been adopted into routine practice. Based on existing evidence, we selected specific morphological features relating to peritumoral inflammatory and stromal responses, agreed criteria for scoring, and assessed these in representative H\&E-stained whole tumour sections, from a population-based cohort of 445 stage II/III colon cancer cases. Moderate/severe peritumoral diffuse lymphoid inflammation and Crohn's disease-like reaction were associated with significantly reduced risks of CRC-specific death (adjusted HR 0.48, 95\% CI 0.31-0.76, and HR 0.60, 95\% CI $0.42-0.84$, respectively). The presence of $>50 \%$ tumour stromal percentage, assessed by global evaluation of tumour area, was associated with a significantly increased risk of CRCspecific death (HR 1.60 95\% CI 1.06-2.41). A composite, “fibroinflammatory score” (0-3), combining dichotomised scores of these three features, showed a highly significant association in relation to survival outcomes. Those with a score $\geq 2$ had an almost 2.5 fold increased risk of CRC-specific death (HR 2.44, 95\% CI: 1.56-3.81), compared with those scoring zero. These associations were stronger in MSI-high tumours, potentially identifying a subset of MSI-high colon cancers which lack characteristic morphological features and have an associated worse prognosis. In summary, reporting on H\&E staining of selected microscopic features of the tumour microenvironment, independently or in combination, offers valuable prognostic information in stage II/III colon cancer and may allow morphological correlation with developing molecular classifications of prognostic and predictive relevance.
\end{abstract}

KEYWORDS: colorectal cancer; stroma; inflammation; prognosis; survival. 


\section{INTRODUCTION}

Colorectal cancer (CRC) is a leading cause of cancer death in developed countries, and is estimated to increase in incidence worldwide, particularly in low-middle income countries. $(1,2)$ Evaluation of tumour morphology has been the mainstay of prognostication for decades, guiding management through assessment of tumour grade, stage and other histological features of proven clinical significance. Recently there has been a major focus on transcription-based molecular classification of CRC and prognostic significance of subtypes. (3-5) However, given the limited availability and current high cost for routine molecular subtyping of CRC, this is likely to remain a future goal for the present time.

Both morphological studies and recent molecular classifications of CRC have highlighted the importance of the tumour microenvironment, reinvigorating interest in the "seed and soil" hypothesis of colorectal carcinogenesis. (6) The first CRC consensus molecular subtype (CMS) classification, which combined gene expression data from over 4000 patients, includes "immune” (CMS1) and "mesenchymal” (CMS4) subtypes. (5) The defining contribution of the tumour microenvironment to the overall expression profile traits associated with each subtype has since been highlighted, in addition to the potential for misclassification due to intratumoral heterogeneity in localised regions of the primary tumour. (7-9) Many prior morphological studies, some decades old, have examined selected features of the tumour microenvironment. Previous assessments by routine microscopy of haematoxylin and eosin (H\&E)-stained sections demonstrated prognostic significance of a variety of features related mainly to peritumoral inflammation and stromal reaction. (10-28) However, there has been limited validation of results in larger studies. Lack of standardisation of methods of assessment and unconvincing interobserver reproducibility for some parameters has prevented uptake into routine reporting practice. 
There is an ongoing need for more reliable and reproducible prognostic morphological features in CRC, especially in stage II disease given well documented divergent clinical outcomes. (29-31) In addition, there is a desire to maximise all available information from routine pathological assessment of CRC resection specimens. To this end, we have chosen from existing literature the most promising tumour microenvironmental features, related to host immune and stromal proliferative responses. We have evaluated these on H\&E-stained whole tumour sections derived from a large population-based cohort of stage II and stage III colon cancer cases. Interobserver and intraobserver reproducibility was rigorously assessed. The overall study aim was to identify those tumour microenvironmental features most likely to provide prognostic information and be feasible to use in routine pathology reporting practice. 


\section{MATERIALS AND METHODS}

\section{Case Identification}

Stage II and III colon cancer patients (ICD-code C.18, $\mathrm{n}=1,862$ ) diagnosed in Northern Ireland from 2004 and up to 2008 were identified from the Northern Ireland Cancer Registry. Rectal and rectosigmoid junction cancers were excluded due to the potential for neoadjuvant therapy to alter tumour morphology. Exclusions were applied to retain only patients with a single, primary colon adenocarcinoma, which had been treated by surgical resection $(n=1,539)$. A detailed clinical case note review was conducted, to verify diagnosis and stage and to extract clinical information including the use of adjuvant chemotherapy and outcome. Following this review, $\mathrm{n}=113$ cases were excluded, mainly on the basis of inaccurate staging. Of the remaining $n=1,426$ patients, $n=740$ patients (52\%) were diagnosed within the jurisdiction of the Northern Ireland Biobank, allowing retrieval of glass slides for pathology review. Slides from $n=661$ (89\%) of these cases were located and, within the study timeframe, $n=473$ consecutive cases underwent slide review and evaluation in this study. These cases were representative of the overall $n=661$ cases with available slides, except they were more likely to be diagnosed in earlier years of the cohort due to the consecutive nature of slide review (data not shown). All patients were followed up for occurrence and cause of death via linkage to the Northern Ireland Registrar General's Office up to $31^{\text {st }}$ December 2013. Patients were recorded as having a CRC specific death if any cause of death was listed as ICD-codes C18, C19, C20 and/or C26. Ethical approval for resection specimen retrieval was received from the Northern Ireland Biobank (REC:11/NI/0013, project NIB13-0069). 


\section{Morphology Review Process}

Generation of the NIB13-0069 study resource included review of all glass slides from the relevant surgical resection specimens to select the two most representative tumour blocks from each case, at least one of which represented the advancing edge/invasive margin of the tumour. Slide review for this study was based on further evaluation of these two representative $H \& E$-stained slides for each case. All cases were reviewed for the selected morphological features by a pathology fellow with an interest in gastrointestinal pathology (SOH). Prior to the study review, random colonic adenocarcinoma cases $(n=10)$ were discussed at two multi-header microscopy conference sessions with three other pathologists, two consultant gastrointestinal pathologists (MBL and PJK) and one trainee pathologist (SI). These four comprised the study pathologist group. The purpose of these sessions was firstly to select suitable morphological features of potential relevance to the study, based on existing evidence, and secondly to decide what criteria to apply to define and score these parameters, applicable to routine practice.

After agreement on study parameters and criteria for scoring, a random set of 36 cases (representing 5\% of the overall 661 cases eligible for inclusion in the study) was chosen for blinded interobserver and intraobserver analysis, the latter following a six week washout period. These analyses involved all observers evaluating both available slides for each case, without any annotations or other indicators of the regions of interest chosen by others.

In addition to the chosen microenvironmental features, the study also assessed tumour typing/subtyping and differentiation, based on World Health Organisation 2010 criteria. (32) Other pathology data items, including nodal status and presence or absence of extramural venous invasion, were recorded from pathology reports. The agreed approach and scoring 
system applied to the study microenvironmental features evaluated is described below and summarised in Table 1.

\section{Peritumoral Lymphoid Inflammation}

The Klintrup-Makinen system, based on H\&E assessment of overall inflammatory response (all inflammatory cell types) at the advancing tumour edge, is widely validated and considered to be reproducible and more valuable than the simpler, preceding Jass scoring system. $(10,13,17,21)$ However, available evidence suggests that, within the inflammatory infiltrates, it is the lymphoid population, assessed on routine microscopy or on immunohistochemistry, which is of most prognostic value. $(12,13,15,17,24)$ Some studies have indicated that immunohistochemical evaluation of specific subsets of lymphoid cell populations currently adds little prognostic value over H\&E assessment. $(12,13,15,33)$ A modification of the Klintrop-Makinen scoring system was therefore chosen, assessing lymphoid cell infiltrates only, within stromal tissue along the invasive tumour front, and ignoring other inflammatory cell populations. Lymphoid infiltrates were scored as absent/mild, featuring scattered lymphocytes only (0); moderate, with a band-like mantle infiltrate and at least focal gland destruction (1); and severe, demonstrating a cup-like lymphoid reaction and marked gland destruction (2). This scoring is illustrated in Figure 1.

\section{Crohn's Disease-like Reaction}

In addition to peritumoral lymphoid reaction at the advancing edge, the so-called Crohn's disease-like reaction (CLR) in peritumoral tissue was also assessed. In contrast to peritumoral diffuse lymphoid inflammation, Crohn's disease-like lymphoid aggregates are comprised 
predominantly of B-cells rather than T-cells. (12) These organised lymphoid structures are an essential component of adaptive immunity. (34) Compared to peritumoral T-cell reaction, relatively little evidence exists for the prognostic value of CLR in CRC. $(12,23,35)$ Scoring was based on the Graham and Appelman criteria, with three categories: none or a maximum of one peritumoral lymphoid aggregate per section (0); occasional lymphoid aggregates with no or rare germinal centres (1); and numerous lymphoid aggregates, some with germinal centres (2). (23) This scoring is illustrated in Figure 2.

\section{Tumour Stromal Percentage}

Multiple studies have reported intratumoral stroma:epithelium ratio or tumour stromal percentage to be prognostic in stage I-III CRC, assessed by different methodologies. $(11,20,22,26)$ Mesker et al simply applied a visual estimation of percentage epithelium and stroma on the whole available tumour area. (20) Two more recent studies employed a focal assessment within a single low-power field from the advancing tumour edge. $(22,26)$ Both of these methods were applied in this study. The global assessment categorised tumours as $>50 \%$ or $<50 \%$ tumour stromal percentage. The focal method involved choosing the most invasive region of the tumour at scanning magnification then, at x10 objective, selecting a field from this region in which tumour epithelium was present at the four cardinal points of the field. A visual estimate of tumour stromal percentage by area was then made from within this single $\mathrm{x} 10$ field, categorised as $<50 \%$ or $>50 \%$. In both the global and focal assessments, empty glandular luminal space, luminal necrosis, extracellular mucin and native smooth muscle tissue were excluded in the visual assessment of tumour stromal percentage. Examples of tumours with high and low stromal percentages are shown in Figure 3. 


\section{Microsatellite Instability Analysis}

DNA was extracted according to the manufacturer's instructions from five $5 \mu \mathrm{m}$ sections of representative tumour blocks using the Maxwell 16 Instrument (Promega, Southampton, UK) and Promega DNA extraction kit, after enrichment for tumour by slide annotation and macrodissection. Quantification of DNA was performed by an absorbance method using the NanoDrop 2000c spectrophotometer (Thermo Scientific, Wilmington, USA). Microsatellite instability (MSI) analysis was performed within the Northern Ireland Molecular Pathology Laboratory according to manufacturer's instructions using the MSI Analysis System, version 1.2 kit (Promega, Southampton, UK) for five mononucleotide repeat markers (BAT-25, BAT-26, NR-21, NR-24 and MONO-27). PCR products were separated by capillary electrophoresis using an ABI 3500 Genetic Analyzer (Fisher Scientific - UK Ltd, Loughborough, UK). The output data was analysed using GeneMapper ${ }^{\circledR}$ v4.1 (Fisher Scientific - UK Ltd, Loughborough, UK) to determine MSI-high (MSI-H) status versus non MSI-H (MSI-low or stable). 


\section{Statistical Analysis}

Of the $n=473$ cases reviewed in this study, patients with a known familial CRC predisposition syndrome $(n=5)$ or who died within the first 30 days of follow-up ( $n=23)$ were excluded, leaving $n=445$ colon cancer patients in our analytical dataset. Kappa values were generated to compare interobserver and intraobserver variability in scoring morphological features. Fleiss's cutoffs indicate poor, fair to good and excellent agreement for kappa scores of $<0.4$, $0.4-0.75$, and $>0.75$, respectively.

Cox proportional hazards analysis was applied to generate hazard ratios (HR) and corresponding 95\% confidence intervals (95\% CI) for overall and CRC-specific survival according to morphological features. Patients were followed up from their date of diagnosis as per cancer registry records until their date of death or $31^{\text {st }}$ December 2013 . Survival analysis was conducted both prior to and after adjustment for potential confounders age, sex, family history of CRC, ECOG performance status, tumour stage, tumour grade, extramural venous invasion and MSI status. Morphological tumour subtype was tested as a confounder but did not alter results, and therefore was not included in the final model. Overall survival analysis was also adjusted for Charlson co-morbidity score.

All morphological features were then entered into a regression model and any that had a $\mathrm{p}<0.25$ were considered to be associated with CRC-specific death - this group comprised peritumoral lymphoid inflammation, CLR and global tumour stromal percentage assessment. Each of these features were then dichotomised so that individuals were assigned a score of 0 or 1 for each whereby 1 = absent/mild peritumoral lymphoid inflammation, absent/mild CLR and $>50 \%$ global tumour stromal percentage (on the basis that these factors were associated with poorer outcomes on individual analysis). Patients could therefore have a combined morphological fibroinflammatory score range of 0-3 (Table 1). 
Stratified analysis for individual morphological features, and combined score, was conducted according to MSI status, chemotherapy receipt and tumour stage. Likelihood ratio tests were applied to formally assess the interaction between these features and CRC survival. All statistical analysis was conducted using Stata version 11.2 (StataCorp, College Station, TX, USA). 


\section{RESULTS}

Characteristics of colorectal cancer patients and tumours

As shown in Table 2, over a mean 5.5 years (range 0.1-10 years) of follow-up, 206 colon cancer patients had died, of which $n=144$ died from a CRC-specific cause. Patients who died from CRC were more likely to be older, have higher ECOG scores (reflecting overall poorer health) and were less likely to receive adjuvant chemotherapy than patients still alive at the end of follow-up. No differences in sex distribution or family history of CRC were observed. In terms of tumour biology, patients who died from CRC were more likely to have higher stage disease, nodal involvement and presence of extramural venous invasion, and less likely to have MSI-high tumours, compared with patients who survived, whereas no differences were seen by WHO tumour type. Similar differences were noted for all-cause deaths.

Interobserver and intraobserver variation for scoring morphological features

Assessment of CLR and tumour stromal percentage by global method demonstrated acceptable levels of interobserver and intraobserver variability (Table 3). Peritumoral lymphoid inflammation demonstrated excellent intraobserver agreement but only poor-fair interobserver agreement. Interobserver and intraobserver agreement on assessment of tumour stromal percentage by focal method was only good at best.

\section{Microenvironmental morphological features and survival}

The two measures of inflammation assessed have some overlap, in that they both looked at peri-tumoural lymphoid reactions. Within the overall patient cohort (of $n=445), 277$ (62.2\%) were scored as 1 or 2 for both peri-tumoural lymphoid inflammation and CLR, while 38 (8.5\%) were scored as 0 for both. Approximately one quarter of patients $(n=115,25.8 \%)$ scored 1 or 2 for peritumoral lymphoid inflammation but 0 for CLR, while 15 (3.4\%) scored 
1 or 2 for CLR but 0 for peritumoral lymphoid inflammation. These proportions were similar regardless of MSI status (data not shown).

As shown in Table 4, the presence of moderate/severe peritumoral diffuse lymphoid inflammation and/or CLR was associated with significant $40-52 \%$ reduced risks of CRC death (adjusted HR 0.48, 95\% CI 0.31-0.76, and HR 0.60, 95\% CI 0.42-0.84, respectively). These associations maintained significance, but were attenuated to $36-37 \%$ reduced risks of death from any cause, suggesting that this good prognostic influence is driven by a CRCspecific effect.

The presence of $>50 \%$ tumour stromal percentage, assessed globally, was associated with an increased risk of CRC death (HR 1.60 95\% CI 1.06-2.41) and death from any cause (HR 1.52, 95\% CI 1.07-2.16). These results were slightly attenuated for CRC-specific survival but remained significant for overall survival after adjustment for other prognostic confounders (Table 4). Tumour stromal percentage assessed focally was not associated with survival outcomes in this cohort.

Combined morphological 'fibroinflammatory' score and survival

The composite score based on dichotomised scores of three features (peritumoral lymphoid inflammation, CLR and tumour stromal percentage) revealed a highly significant association when analysing this score in relation to survival outcomes, even after adjustment for known confounders including differentiation (Table 5, Figure 4). Patients with a score of one had a 53\% increase in the hazard for CRC-specific death (HR 1.53, 95\% CI: 1.02-2.28), and those with a score $\geq 2$ had an almost 2.5 fold increased risk of CRC-specific death (HR 2.44, 95\% CI: 1.56-3.81), compared with those scoring zero. Similar results were seen for all cause mortality. 
Figure 5 illustrates similar directions of prognostic influence of the morphological features in both MSI status groups, although the magnitude of associations was considerably stronger in the MSI-high group. Patients with a fibroinflammatory score of 2 or 3, in comparison with those having a score of 0 , had a 66\% increased risk of CRC-death in the non MSI-high group, compared to a six-fold increased risk of CRC-death in the MSI-high group, although the latter is based on a very small number of patients ( $\mathrm{n}=8$ patients had MSI-H tumours with a fibroinflammatory score of 2 or 3, and seven of these patients died during the follow-up period). A significant interaction was observed between CRC survival, MSI status and peritumoral inflammatory infiltrate $(\mathrm{p}=0.002)$, and the overall fibroinflammatory score $(\mathrm{p}=0.01)$.

Subgroup analysis by adjuvant chemotherapy receipt and tumour stage revealed largely similar results to those seen in the overall cohort of CRC patients, although the magnitude of associations for inflammatory features was slightly stronger in stage III compared with stage II patients (Supplementary Table 1). 


\section{DISCUSSION}

The results from this large population-based cohort of stage II and III colon cancer patients indicate that three tumour microenvironmental features not currently reported in routine practice - peritumoral lymphoid infiltrate, CLR and global assessment of tumour stromal percentage - are predictive of survival outcomes. These associations were evident when taking into consideration other established prognostic factors, such as tumour grade, stage, extramural venous invasion and MSI status, and patient demographics. With some caveats, assessment of these features has acceptable interobserver and intraobserver variation, suggesting that their introduction into routine clinical practice is feasible.

Despite significant progress in the molecular understanding of colorectal carcinogenesis, routine pathology reporting remains largely based on microscopic tumour assessment. Furthermore, reporting is focussed almost exclusively on the epithelial tumour component, for example in staging and grading, and the tumour microenvironment is largely ignored. $(36,37)$ This is despite numerous morphological studies demonstrating prognostic significance of selected tumour microenvironmental features (10-28). Broadly, these have related either to peritumoral inflammatory response or to the intratumoral stromal component.

Peritumoral inflammation in CRC has been the subject of a large number of studies, both morphological and immunohistochemical, and recently a proposal has been advanced to report an Immunoscore for all CRC cases, applying immunohistochemistry and digital image analysis for scoring. (24) The presence of a significant peritumoral inflammatory infiltrate has assumed increased potential relevance recently for therapy-related decisions, with the advent of immune-based therapies including checkpoint CTLA-4, PD-1 and PDL-1 inhibitors such as ipilimumab which are currently being investigated in trials in colorectal and other cancers. (38) For example, we recently highlighted the presence of a good prognostic group 
in stage III CRC defined by high PD-L1 gene expression within the peritumoral immune cell infiltrate. (39)

Routine application of multiple biomarkers by immunohistochemistry and digital image analysis for scoring remains unfeasible in routine reporting practice however, particularly within low-middle income countries where CRC incidence is rising most, and so more consideration of routine H\&E assessment is required. Regarding peritumoral inflammatory composition, the lymphoid component appears to offer most prognostic information, beyond the overall peritumoral inflammatory assessment favoured by Klintrop-Makinen, or other selected inflammatory cell populations, and so peritumoral lymphoid inflammation was the chosen parameter in this study. Although interobserver variation in assessment was only poor-fair, post-study discussion by the four study pathologists suggested this was in part related to the handling of certain inflammatory sub-populations e.g. plasma cells. This highlights the importance of meticulous attention to standardisation of assessment criteria and agreement of definitions. Intraobserver variation was excellent and previous studies have also indicated good reproducibility for assessment of peritumoral inflammatory cell populations on H\&E examination. (16,21) Nevertheless, reproducibility of such assessments is of paramount importance and requires rigorous evaluation in all such morphological studies and further careful consideration prior to incorporation of such assessments into routine practice. Whether evaluation of specific peritumoral inflammatory cell populations is preferably done by $H \& E$ examination or by immunohistochemistry, in addition requires consideration of resource and prognostic or predictive clinical application.

Both peritumoral lymphoid inflammation and CLR demonstrated a protective prognostic effect in this study, and of similar magnitudes. This supports numerous previous studies of peritumoral diffuse inflammation, based on morphological and immunohistochemical assessments. $(12,13,15,17,24)$ Less literature exists on the prognostic benefit of CLR in CRC, 
although one study, by Graham and Appelman, dates from 1990. (23) A recent, large study of CRC stages I-IV, reported prognostic value of peritumoral lymphoid aggregates that was independent of stage and of peritumoral inflammatory assessment (assessed by KlintrupMakinen score). (13)

There was considerable overlap in scoring patterns for peritumoral lymphoid inflammation and CLR. Of the approximately 30\% of patients in the cohort having differential scoring for these two inflammatory parameters, most of these discordant cases scored 1 or 2 for peritumoral inflammation and 0 for CLR. A smaller proportion of patients $3.4 \%$, $(n=15)$ conversely scored 1 or 2 for CLR and 0 for peritumoral lymphoid inflammation. Importantly, in the present study reproducibility of CLR assessments was significantly better than for peritumoral lymphoid inflammation. Thus we consider both worthy of independent assessment, in accordance with the study by Klintrup et al, which concluded that CLR and inflammatory reaction at the advancing tumour edge do not represent a phenotypic variation of the same immune defence mechanism, but rather are independent and each of prognostic value. (21)

We adopted two approaches to assessing tumour stromal percentage, one global and one focal, both reported previously to have prognostic relevance in CRC. $(20,22,26)$ West et al used a more precise morphometric method, and demonstrated similar findings, but this approach is considered too time-consuming for routine use, taking on average 20 minutes per case in that study. (11) We found the global assessment of $>50 \%$ tumour stromal percentage, but not the focal assessment, to be associated with significantly increased hazard for a CRCspecific cause of death. Both intraobserver and interobserver reproducibility of global stromal assessment was also much better than of focal stromal assessment. This contradicts findings from two previous studies, both describing excellent reproducibility of the focal method. $(22,26)$ However, while our study allowed each pathologist to select an independent study 
field based on their own assessment of the requirements, neither of these papers describes in sufficient detail how interobserver variability was assessed, specifically how independent was selection of study fields between observers. On post-study evaluation, we found selection of "the most invasive field", required by the Huijbers et al method, varied considerably between study participants, even when examining only two slides per case, and this had a profound influence on reproducibility. This can only be expected to be a greater problem in everyday reporting practice, with more tumour slides to evaluate per case. Global assessment, considering all available tumour tissue area, as originally proposed by Mesker et al in a smaller study, appears to be a more reproducible and valuable parameter.(20)

Combining dichotomised scores from the three tumour microenvironmental features found to be of prognostic value, a 'fibroinflammatory score' (0-3) was derived for each case, and was found to better predict prognosis than any of the individual features, with a highly significant association evident. One previous study has assessed similar tumour microenvironmental features in a large CRC cohort, and described tumour stromal prognostic influence in the context of local and systemic host responses, including peritumoral inflammation, but, to our knowledge, this is the first study to combine these features to derive a tumour microenvironmental score of local stromal and inflammatory response. (26) The potential for confounding interobserver variation in assessments of individual features can be reduced by using the proposed combined score.

This proposed addition to routine morphological assessment of CRC correlates well with renewed interest in tumour microenvironment, and the concept of distinguishing features of the tumour "seed” (malignant epithelium) from tumour "soil” (immune system and stroma). (6) This impacts on proposed transcriptional profiling of CRC, with CMS subtypes including an "immune” subtype (CMS1), characterised largely by microsatellite instability and related clinicopathological features including excellent prognosis, and a "mesenchymal" subtype 
(CMS4), characterised by microsatellite stability, epithelial-mesenchymal transition and poor prognosis. (5) Sub-analysis by MSI status in our cohort suggests that, within the group of MSI-H colon cancers, there exists a sub-group which lacks the characteristic morphological features (scoring 2 or 3 for fibroinflammatory score), and this group has a worse prognosis than MSI-H tumours with classical morphology. Therefore, morphological assessment of the tumour microenvironment adds value beyond MSI status to prognostication in the MSI-H group in particular. This is in agreement with our findings from a transcription-based study, where some MSI-H tumours appeared to have a low immune infiltration signature similar to non-MSI high tumours. (39) Additionally, these findings support a recent study indicating that tumour Immunoscore offers greater prognostic value than assessment of MSI status. (40)

The strengths of this study include its population-representative cohort of stage II and III colon cancer patients, relatively large overall study numbers, careful consideration of reproducibility of assessments and feasibility for introduction into routine practice and correlation of traditional morphological assessment with current molecular approaches to CRC subtyping. A study weakness is that restriction of tumour assessments to two slides per case, rather than examining all tumour slides, does not reflect real life practice and may distort somewhat data on reproducibility. Also, we have not examined rectal cancers in this study.

In summary, supporting previous studies, we have found that reporting of selected features of the tumour microenvironment in stage II/III colon cancer, offers prognostic information. We consider a combination of peritumoral lymphoid inflammation at the advancing edge, Crohn's disease-like reaction and tumour stromal percentage as the microenvironmental features likely to be most reproducibly assessed and offer the most prognostic value. We propose therefore that assessment of these tumour microenvironmental parameters be 
introduced into routine reporting practice, at least until such times that adjunctive targeted immunohistochemistry panels and digital image analysis are more readily available. 


\section{ACKNOWLEDGEMENTS}

Dr. Seán Hynes has been supported through the Dr. Richard Stevens Fellowship awarded by the Health Service Executive, Ireland. Dr. Helen Coleman is a co-investigator of the UKCRC Centre of Excellence for Public Health Northern Ireland. Drs. Maurice Loughrey and Paul Kelly receive funding from Cancer Research UK (CR-UK) through the Belfast CRUK Centre initiative. The samples used in this research were received from the Northern Ireland Biobank which is funded by Health and Social Care Research and Development Division of the Public Health Agency in Northern Ireland and CR-UK through the Belfast CR-UK Centre and the Northern Ireland Experimental Cancer Medicine Centre; additional support was received from the Friends of the Cancer Centre. The Northern Ireland Molecular Pathology Laboratory, which is responsible for creating resources for the Northern Ireland Biobank, has received funding from CR-UK, the Friends of the Cancer Centre and the Sean Crummey Foundation. The Northern Ireland Cancer Registry is funded by the Public Health Agency, Northern Ireland.

Author contributions: All authors have contributed to the intellectual content of this paper and have made significant contributions to the conception and design, drafting or revising the article for intellectual content; and final approval of the published article. 


\section{References}

1. Arnold M, Sierra MS, Laversanne M, et al. Global patterns and trends in colorectal cancer incidence and mortality. Gut 2016; DOI: 10.1136/gutjnl-2015-310912.

2. Torre LA, Bray F, Siegel RL, et al. Global cancer statistics, 2012. CA Cancer J Clin 2015; 65: 87-108.

3. Sinicrope FA, Shi Q, Smyrk TC, et al. Molecular markers identify subtypes of stage III colon cancer associated with patient outcomes. Gastroenterology 2015; 148: 88-99.

4. Phipps AI, Limburg PJ, Baron JA, et al. Association between molecular subtypes of colorectal cancer and patient survival. Gastroenterology 2015; 148: 77-87.

5. Guinney J, Dienstmann R, Wang X, et al. The consensus molecular subtypes of colorectal cancer. Nat Med 2015; 21: 1350-1356.

6. Seretis F, Seretis C, Youssef H, et al. Colorectal cancer: seed and soil hypothesis revisited. Anticancer Res 2014; 34: 2087-2094.

7. Calon A, Lonardo E, Berenguer-Llergo A, et al. Stromal gene expression defines poorprognosis subtypes in colorectal cancer. Nat Genet 2015; 47: 320-329.

8. Dunne PD, McArt DG, Bradley CA, et al. Challenging the cancer molecular stratification dogma: Intratumoral heterogeneity undermines consensus molecular subtypes and potential diagnostic value in colorectal cancer. Clin Cancer Res 2016; 22: 4095-104.

9. Isella C, Terrasi A, Bellomo SE, et al. Stromal contribution to the colorectal cancer transcriptome. Nat Genet 2015; 47: 312-319. 
10. Jass JR, Love SB, Northover JM. A new prognostic classification of rectal cancer. Lancet 1987; 1: 1303-1306.

11. West NP, Dattani M, McShane P, et al. The proportion of tumour cells is an independent predictor for survival in colorectal cancer patients. Br J Cancer 2010; 102: 1519-1523.

12. Vayrynen JP, Sajanti SA, Klintrup K, et al. Characteristics and significance of colorectal cancer associated lymphoid reaction. Int J Cancer 2014; 134: 2126-2135.

13. Vayrynen JP, Tuomisto A, Klintrup K, et al. Detailed analysis of inflammatory cell infiltration in colorectal cancer. Br J Cancer 2013; 109: 1839-1847.

14. Ueno H, Shinto E, Shimazaki H, et al. Histologic categorization of desmoplastic reaction: its relevance to the colorectal cancer microenvironment and prognosis. Ann Surg Oncol 2015; 22: $1504-1512$.

15. Roxburgh CS, McMillan DC. The role of the in situ local inflammatory response in predicting recurrence and survival in patients with primary operable colorectal cancer. Cancer Treat Rev 2012; 38: 451-466.

16. Roxburgh CS, Salmond JM, Horgan PG, et al. Tumour inflammatory infiltrate predicts survival following curative resection for node-negative colorectal cancer. Eur J Cancer 2009; 45: $2138-2145$.

17. Richards CH, Flegg KM, Roxburgh CS, et al. The relationships between cellular components of the peritumoral inflammatory response, clinicopathological characteristics and survival in patients with primary operable colorectal cancer. Br J Cancer 2012; 106: 20102015. 
18. Pages F, Kirilovsky A, Mlecnik B, et al. In situ cytotoxic and memory T cells predict outcome in patients with early-stage colorectal cancer. J Clin Oncol 2009; 27: 5944-5951.

19. Ogino S, Nosho K, Irahara N, et al. Lymphocytic reaction to colorectal cancer is associated with longer survival, independent of lymph node count, microsatellite instability, and CpG island methylator phenotype. Clin Cancer Res 2009; 15: 6412-6420.

20. Mesker WE, Junggeburt JM, Szuhai K, et al. The carcinoma-stromal ratio of colon carcinoma is an independent factor for survival compared to lymph node status and tumor stage. Cell Oncol 2007; 29: 387-398.

21. Klintrup K, Makinen JM, Kauppila S, et al. Inflammation and prognosis in colorectal cancer. Eur J Cancer 2005; 41: 2645-2654.

22. Huijbers A, Tollenaar RA, v Pelt GW, et al. The proportion of tumor-stroma as a strong prognosticator for stage II and III colon cancer patients: validation in the VICTOR trial. Ann Oncol 2013; 24: 179-185.

23. Graham DM, Appelman HD. Crohn's-like lymphoid reaction and colorectal carcinoma: a potential histologic prognosticator. Mod Pathol 1990; 3: 332-335.

24. Galon J, Mlecnik B, Bindea G, et al. Towards the introduction of the 'Immunoscore' in the classification of malignant tumours. J Pathol 2014; 232: 199-209.

25. Ropponen KM, Eskelinen MJ, Lipponen PK, et al. Prognostic value of tumour-infiltrating lymphocytes (TILs) in colorectal cancer. J Pathol 1997; 182: 318-324. 
26. Park JH, Richards $\mathrm{CH}$, McMillan DC, et al. The relationship between tumour stroma percentage, the tumour microenvironment and survival in patients with primary operable colorectal cancer. Ann Oncol 2014; 25: 644-651.

27. Pages F, Berger A, Camus M, et al. Effector memory T cells, early metastasis, and survival in colorectal cancer. $N$ Engl $J$ Med 2005; 353: 2654-2666.

28. Fridman WH, Galon J, Pages F, et al. Prognostic and predictive impact of intra- and peritumoral immune infiltrates. Cancer Res 2011; 71: 5601-5605.

29. Quasar Collaborative Group, Gray R, Barnwell J, et al. Adjuvant chemotherapy versus observation in patients with colorectal cancer: a randomised study. Lancet 2007; 370: 20202029.

30. Kennedy RD, Bylesjo M, Kerr P, et al. Development and independent validation of a prognostic assay for stage II colon cancer using formalin-fixed paraffin-embedded tissue. $J$ Clin Oncol 2011; 29: 4620-4626.

31. Niedzwiecki D, Frankel WL, Venook AP, et al. Association Between Results of a Gene Expression Signature Assay and Recurrence-Free Interval in Patients With Stage II Colon Cancer in Cancer and Leukemia Group B 9581 (Alliance). J Clin Oncol 2016; 34: 30473053.

32. Hamilton SR, Bosman FT, Boffetta P, et al. Carcinoma of the colon and rectum. In: Bosman FT, Carneiro F, Hruban RH, Theise ND, eds. WHO classification of tumours of the digestive system. Lyon: IARC Press; 2010.

33. Mei Z, Liu Y, Liu C, et al. Tumour-infiltrating inflammation and prognosis in colorectal cancer: systematic review and meta-analysis. Br J Cancer 2014; 110: 1595-1605. 
34. Cyster JG. Lymphoid organ development and cell migration. Immunol Rev 2003; 195: 514.

35. Rozek LS, Schmit SL, Greenson JK, et al. Tumor-Infiltrating Lymphocytes, Crohn's-Like Lymphoid Reaction, and Survival From Colorectal Cancer. J Natl Cancer Inst 2016; 108: (8). DOI: 10.1093/jnci/djw027.

36. Loughrey MB, Quirke P, Shepherd NA. Standards and datasets for reporting cancers: Dataset for colorectal cancer histopathology reports. London: Royal College of Pathologists; 2014.

37. Tang LK, Berlin J, Branton P, et al. College of american pathologists: protocol for the examination of specimens from patients with primary carcinoma of the colon and rectum. 2016. Accessed November 1st, 2016.

38. Moehler M, Delic M, Goepfert K, et al. Immunotherapy in gastrointestinal cancer: Recent results, current studies and future perspectives. Eur J Cancer 2016; 59: 160-170.

39. Dunne PD, McArt DG, O'Reilly PG, et al. Immune-Derived PD-L1 Gene Expression Defines a Subgroup of Stage II/III Colorectal Cancer Patients with Favorable Prognosis Who May Be Harmed by Adjuvant Chemotherapy. Cancer Immunol Res 2016; 4: 582-591.

40. Mlecnik B, Bindea G, Angell HK, et al. Integrative Analyses of Colorectal Cancer Show Immunoscore Is a Stronger Predictor of Patient Survival Than Microsatellite Instability. Immunity 2016; 44: 698-711. 


\section{FIGURES}

Figure 1. Examples of peritumoral lymphoid infiltrate scoring: score 0, absent/mild, featuring scattered lymphocytes only (A, x100); score 1, moderate, with a band-like mantle infiltrate and at least focal gland destruction (B, x100); score 2, severe, demonstrating a cuplike lymphoid reaction and marked gland destruction (C, x100).

Figure 2. Examples of Crohn's disease-like reaction scoring: score 0, none or a maximum of one peritumoral lymphoid aggregate (A, x40); score 1, occasional lymphoid aggregates with no or rare germinal centres $(\mathrm{B}, \mathrm{x} 40)$; score 2, numerous lymphoid aggregates, some with germinal centres (C, x40).

Figure 3. Examples of colon cancers with less than $50 \%(A, x 40)$ and greater than $(B, x 40)$ tumour stromal percentage.

Figure 4. Kaplan Meier graph showing colorectal cancer-specific survival amongst stage II and III colon cancer patients according to combined fibroinflammatory score.

Figure 5. Risk of colorectal cancer-specific death according to tumour microenvironmental features in stage II and III colon cancer cases, stratified by microsatellite instability status. 
Table 1. Approach to scoring of histological features.

\begin{tabular}{|c|c|c|c|}
\hline Feature & Approach to scoring & Scoring categories & References \\
\hline $\begin{array}{l}\text { Peritumoral } \\
\text { inflammation }\end{array}$ & $\begin{array}{l}\text { Restricted to lymphoid infiltrates only, ignoring other } \\
\text { inflammatory cell populations, and assessed along the } \\
\text { invasive front of the tumour. }\end{array}$ & $\begin{array}{l}0=\text { absent } / \text { mild (scattered lymphoid cells only) } \\
1=\text { moderate }, \text { a band-like infiltrate and focal gland destruction } \\
2=\text { severe, a cup-like lymphoid reaction and marked gland } \\
\text { destruction. }\end{array}$ & $(21)$ \\
\hline $\begin{array}{l}\text { Crohn's disease- } \\
\text { like lymphoid } \\
\text { reaction }\end{array}$ & $\begin{array}{l}\text { Assessed along the invasive front of the tumour, ignoring } \\
\text { normal mucosal/submucosal lymphoid aggregates. }\end{array}$ & $\begin{array}{l}0=0-1 \text { peritumoral lymphoid aggregate per section } \\
1=\text { occasional lymphoid aggregates with no or rare germinal centres } \\
2=\text { numerous lymphoid aggregates, some with germinal centres }\end{array}$ & (23) \\
\hline $\begin{array}{l}\text { Tumour stromal } \\
\text { percentage (focal) }\end{array}$ & $\begin{array}{l}\text { The most invasive region of the tumour was selected at } \\
\text { scanning magnification then, at } 10 x \text { objective, a field was } \\
\text { selected from this region in which tumour was present at the } \\
\text { four cardinal points. A visual estimate of tumour stromal } \\
\text { percentage by area was then made from within this single } \\
\text { x10 field. Empty glandular luminal spaces, luminal necrosis, } \\
\text { extracellular mucin and native smooth muscle tissue were } \\
\text { excluded in this assessment. }\end{array}$ & $\begin{array}{l}0=<50 \% \text { tumour stromal percentage } \\
1=>50 \% \text { tumour stromal percentage }\end{array}$ & $(22,26)$ \\
\hline $\begin{array}{l}\text { Tumour stromal } \\
\text { percentage } \\
\text { (global) }\end{array}$ & $\begin{array}{l}\text { At scanning magnification, the overall tumour stromal } \\
\text { percentage was estimated. Empty glandular luminal spaces, } \\
\text { luminal necrosis, extracellular mucin and native smooth } \\
\text { muscle tissue were excluded in this assessment. }\end{array}$ & $\begin{array}{l}\text { A visual estimate of tumour stromal percentage, expressed as the } \\
\text { total area of tumour stroma plus epithelium, was categorised as: } \\
0=<50 \% \text { stroma } \\
1=>50 \% \text { stroma }\end{array}$ & (20) \\
\hline $\begin{array}{l}\text { Combined } \\
\text { fibroinflammatory } \\
\text { score }\end{array}$ & $\begin{array}{l}\text { Three of the above features, selected on the basis of those } \\
\text { associated with poorest outcomes on individual analysis, } \\
\text { were dichotomised so that individuals were assigned a score } \\
\text { of } 0 \text { or } 1 \text { for each. Patients could therefore have a combined } \\
\text { morphological fibroinflammatory score range of } 0-3 \text {. }\end{array}$ & $\begin{array}{l}\text { Combined fibroinflammatory score, totalled from above features } \\
\text { whereby: } \\
1=\text { absent/mild peritumoral lymphoid inflammation } \\
1=\text { absent/mild Crohn's disease-like lymphoid reaction } \\
1=>50 \% \text { global tumour stromal percentage }\end{array}$ & \\
\hline
\end{tabular}


Table 2. Descriptive characteristics of colon cancer cases by vital status at end of follow-up period.

\begin{tabular}{|c|c|c|c|c|}
\hline Characteristic & $\begin{array}{l}\text { Total } \\
\mathrm{n}=445(\%)\end{array}$ & $\begin{array}{l}\text { Alive } \\
\mathrm{n}=239(\%)\end{array}$ & $\begin{array}{l}\text { Death } \\
\mathrm{n}=206(\%)\end{array}$ & $\begin{array}{l}\text { CRC death } \\
n=144(\%)\end{array}$ \\
\hline \multicolumn{5}{|l|}{ Sex } \\
\hline Male & $237(53.3)$ & $126(52.7)$ & 111 (53.9) & 82 (56.9) \\
\hline Female & 208 (46.7) & $113(47.3)$ & 95 (46.1) & $62(43.1)$ \\
\hline \multicolumn{5}{|l|}{ Age, years } \\
\hline Median (IQR) & $73(65-79)$ & $70(63-76)$ & $76(69-81)$ & $78(74-83)$ \\
\hline$<50$ & $20(4.5)$ & $13(5.4)$ & $7(3.4)$ & $6(4.2)$ \\
\hline $50-<60$ & $39(8.8)$ & $28(11.7)$ & $11(5.3)$ & $9 \quad(6.3)$ \\
\hline $60-<70$ & $121(27.2)$ & 79 (33.1) & $42(20.4)$ & $37(25.7)$ \\
\hline $70-<80$ & $175(39.3)$ & $90(37.7)$ & $85(41.3)$ & $54(37.5)$ \\
\hline$\geq 80$ & $90(20.2)$ & $29(12.1)$ & $61(29.6)$ & $38(26.4)$ \\
\hline \multicolumn{5}{|l|}{ Year of diagnosis } \\
\hline 2004 & $95 \quad(21.4)$ & $41(17.2)$ & $54(26.2)$ & $35(24.3)$ \\
\hline 2005 & $117(26.3)$ & $59(24.7)$ & $58(28.2)$ & 43 (29.9) \\
\hline 2006 & $78 \quad(17.5)$ & $33(13.8)$ & $45(21.8)$ & 33 (22.9) \\
\hline 2007 & $81 \quad(18.2)$ & $52(21.8)$ & $29(14.1)$ & $17(11.8)$ \\
\hline 2008 & $74 \quad(16.6)$ & $54(22.6)$ & $20(9.7)$ & $16(11.1)$ \\
\hline \multicolumn{5}{|l|}{ ECOG status } \\
\hline $0-1$ & $237(53.3)$ & $138(57.7)$ & $99(48.1)$ & $65(45.1)$ \\
\hline 2 & $30(6.7)$ & $11(4.6)$ & $19(9.2)$ & $15(10.4)$ \\
\hline $3-4$ & $17(3.8)$ & $4 \quad(1.8)$ & $13(6.3)$ & $10(6.9)$ \\
\hline Unknown & $161(36.2)$ & $86(36.0)$ & $75(36.4)$ & $54(37.5)$ \\
\hline \multicolumn{5}{|c|}{ Charlson co-morbidity score } \\
\hline Median (IQR) & $0(0-1)$ & $0(0-1)$ & $0(0-1)$ & $0(0-1)$ \\
\hline \multicolumn{5}{|l|}{ Family history CRC } \\
\hline No & $227(51.0)$ & $128(53.6)$ & 99 (48.1) & 69 (47.9) \\
\hline Yes & $54 \quad(12.1)$ & 33 (13.8) & $21(10.2)$ & $19(13.2)$ \\
\hline Unknown & $164(36.9)$ & 78 (32.6) & $86(41.7)$ & 56 (38.9) \\
\hline \multicolumn{5}{|c|}{ Adjuvant chemotherapy use } \\
\hline No & 320 (71.9) & $157(65.7)$ & $163(79.1)$ & 105 (72.9) \\
\hline Yes & $125(28.1)$ & 82 (34.3) & 43 (20.9) & 39 (27.1) \\
\hline \multicolumn{5}{|l|}{ Stage } \\
\hline$\|$ & $275(61.8)$ & $160(70.0)$ & $115(55.8)$ & $65(45.1)$ \\
\hline III & $170(38.2)$ & 79 (33.0) & 91 (44.2) & $79(54.9)$ \\
\hline \multicolumn{5}{|l|}{ Nodal status } \\
\hline NO & $275(61.8)$ & $160(70.0)$ & $115(55.8)$ & $65(45.1)$ \\
\hline N1 & $110(24.7)$ & 54 (22.6) & $56(27.2)$ & $47(32.6)$ \\
\hline N2 & $60 \quad(13.5)$ & $25(10.4)$ & $35(17.0)$ & $32(22.2)$ \\
\hline \multicolumn{5}{|l|}{ Differentiation } \\
\hline Well/moderate & $408(91.7)$ & $223(93.3)$ & $185(89.8)$ & $129(89.6)$ \\
\hline Poor & $37(8.3)$ & $16(6.7)$ & $21(10.2)$ & $15(10.4)$ \\
\hline \multicolumn{5}{|l|}{ Extramural venous invasion } \\
\hline No & $293(65.8)$ & $173(72.4)$ & $120(58.3)$ & $80(55.6)$ \\
\hline Yes & $108(24.3)$ & $48 \quad(20.1)$ & $60(29.1)$ & $50(34.7)$ \\
\hline Unknown & 44 (9.9) & $18(7.5)$ & $26(12.6)$ & $14(9.7)$ \\
\hline \multicolumn{5}{|l|}{ Tumor (sub)type } \\
\hline Adenocarcinoma, NOS & $412(92.6)$ & $222(92.9)$ & $190(92.2)$ & $131(91.0)$ \\
\hline Other* & $33(7.4)$ & $17(7.1)$ & $16(7.8)$ & $13(9.0)$ \\
\hline
\end{tabular}


MSI Status

Non MSI-high

309 (70.2) $164(69.8)$

145 (70.7)

$107(74.8)$

MSI-high

99 (22.5)

$60 \quad(25.5)$

$39 \quad(19.0)$

$22(15.4)$

Unknown

$32(7.3)$

11 (4.7)

21 (10.2)

$14(9.8)$

Table 2 legend.

CRC death: Colorectal cancer-specific death; ECOG: Eastern Cooperative Oncology Group performance status of cancer patients' general well-being and activities of daily life, 0-1: fully active or restricted strenuous activity but able to carry out light activities, 2: ambulatory and able to selfcare, unable to carry out work activities, 3-4: limited or no self-care, or bedbound; NOS: not otherwise specified; MSI: microsatellite instability. IQR: Inter-quartile range.

*All tumours were coded by cancer registry as adenocarcinoma. Following slide review in this study, specific types/subtypes identified other than adenocarcinoma, NOS, comprised mucinous adenocarcinoma $(n=29)$, medullary carcinoma $(n=3)$ and signet ring cell adenocarcinoma $(n=1)$. 
Table 3. Kappa values reflecting inter-observer and intra-observer variation in assessment of morphological features between four pathologists.

\begin{tabular}{|l|l|lll|l|}
\hline & Pathologist & 1 & 2 & 3 & Judgement on variation \\
\hline Peritumoral & 4 & 0.48 & 0.30 & 0.16 & Interobserver: poor-fair \\
inflammation & 3 & 0.05 & 0.30 & & \\
(0 v. 1/2) & 2 & 0.44 & & & \\
& $1^{*}$ & 0.79 & & & Intraobserver: excellent \\
\hline Crohn's disease-like & 4 & 0.84 & 0.58 & 0.46 & Interobserver: poor-excellent \\
lymphoid reaction & 3 & 0.47 & 0.29 & & \\
(0 v. 1/2) & 2 & 0.62 & & & \\
& $1^{*}$ & 0.84 & & & Intraobserver: excellent \\
\hline Tumor stromal & 4 & 0.53 & 0.49 & 0.22 & Interobserver: poor-good \\
percentage (focal) & 3 & 0.13 & 0.43 & & \\
(<50\% v. >50\%) & 2 & 0.21 & & & \\
& $1^{*}$ & 0.45 & & & Intraobserver: fair-good \\
\hline Tumor stromal & 4 & 1.00 & 0.55 & 0.48 & Interobserver: good-excellent \\
percentage (global) & 3 & 0.48 & 0.75 & & \\
(<50\% v. >50\%) & 2 & 0.55 & & & \\
& $1^{*}$ & 0.84 & & & Intraobserver: excellent \\
\hline
\end{tabular}

*Intra-observer variation kappa from same pathologist after a six week wash-out period. 
Table 4. Risk of colorectal cancer-specific or overall death according to morphological features in stage II and III colon cancer cases.

\begin{tabular}{|c|c|c|c|c|c|c|c|}
\hline Morphological feature & $\begin{array}{l}\text { Alive } \\
\mathrm{n}=239(\%)\end{array}$ & \multicolumn{3}{|c|}{ Colorectal cancer-specific mortality } & \multicolumn{3}{|c|}{ All cause mortality } \\
\hline \multicolumn{8}{|c|}{ Peritumoral lymphoid inflammation } \\
\hline $1 / 2$ & $217(90.8)$ & $117(81.3)$ & $0.49(0.32-0.75)$ & $0.48(0.31-0.76)$ & $175(84.9)$ & $0.63(0.43-0.92)$ & $0.63(0.42-0.95)$ \\
\hline \multicolumn{8}{|c|}{ Crohn's disease-like reaction } \\
\hline 0 & $67(28.0)$ & $65(45.1)$ & 1.00 & 1.00 & $86 \quad(41.8)$ & 1.00 & 1.00 \\
\hline $1 / 2$ & $172(72.0)$ & 79 (54.9) & $0.54(0.39-0.74)$ & $0.60(0.42-0.84)$ & $120(58.2)$ & $0.61(0.47-0.81)$ & $0.64(0.48-0.86)$ \\
\hline$>50 \%$ & $48(20.1)$ & $36(25.0)$ & $1.28(0.88-1.87)$ & $1.14(0.75-1.72)$ & $51(24.8)$ & $1.27(0.93-1.75)$ & $1.23(0.87-1.73)$ \\
\hline \multicolumn{8}{|l|}{ Tumor stromal \% (global) } \\
\hline$<50 \%$ & $212(88.7)$ & $116(80.6)$ & 1.00 & 1.00 & $168(81.6)$ & 1.00 & 1.00 \\
\hline$>50 \%$ & $27(11.3)$ & $28(19.4)$ & $1.60(1.06-2.41)$ & $1.45(0.92-2.29)$ & $38(18.4)$ & $1.52(1.07-2.16)$ & $1.49(1.02-2.20)$ \\
\hline
\end{tabular}

$\mathrm{Cl}$ : Confidence Intervals; CRC: Colorectal cancer; HR: Hazard ratio.

*Adjusted for: sex (male/female), adjuvant chemotherapy use (yes/no), age ( $<50,50-<60,60-<70,70-<80$, $\geq 80$ years), year of diagnosis (2004-2008), stage (II/III), family history of colorectal cancer (yes/no/unknown), MSI status (non MSI-high/MSI-high/unknown), extramural venous invasion (no/yes/unknown), and ECOG status (0-1/2/3-4/unknown), differentiation (well-moderate/poor) and (for all-cause mortality analysis only) Charlson co-morbidity score (0-7). 
Table 5. Combined score of fibroinflammatory morphological features and risk of colorectal cancer-specific or overall death in stage II and III colon cancer cases.

\begin{tabular}{|c|c|c|c|c|c|c|c|}
\hline \multirow[b]{2}{*}{$\begin{array}{l}\text { Combined } \\
\text { fibroinflammatory } \\
\text { index score* }\end{array}$} & \multirow[b]{2}{*}{$\begin{array}{l}\text { Alive } \\
\mathrm{n}=239(\%)\end{array}$} & \multirow[b]{2}{*}{$\begin{array}{l}\text { CRC death } \\
n=144(\%)\end{array}$} & \multicolumn{2}{|c|}{ CRC-specific mortality } & \multirow[b]{2}{*}{$\begin{array}{l}\text { Death } \\
n=206 \text { (\%) }\end{array}$} & \multicolumn{2}{|c|}{ All-cause mortality } \\
\hline & & & $\begin{array}{l}\text { Unadjusted HR } \\
(95 \% \mathrm{Cl})\end{array}$ & $\begin{array}{l}\text { Adjusted HR** } \\
(95 \% \mathrm{Cl})\end{array}$ & & $\begin{array}{l}\text { Unadjusted HR } \\
(95 \% \mathrm{Cl})\end{array}$ & $\begin{array}{l}\text { Adjusted HR** } \\
(95 \% \mathrm{Cl})\end{array}$ \\
\hline 0 & $150(62.8)$ & $61(42.4)$ & 1.00 & 1.00 & $97(47.1)$ & 1.00 & 1.00 \\
\hline 1 & $65 \quad(27.2)$ & $49(34.0)$ & $1.75(1.20-2.54)$ & $1.53(1.02-2.28)$ & $67(32.5)$ & $1.52(1.12-2.08)$ & $1.46(1.05-2.04)$ \\
\hline $2-3$ & $24(10.0)$ & 35 (23.6) & $2.65(1.74-4.03)$ & $2.44(1.56-3.81)$ & $42(20.4)$ & 2.08 (1.45-2.99) & $2.00(1.36-2.95)$ \\
\hline
\end{tabular}

$\mathrm{Cl}$ : Confidence intervals; CRC: Colorectal cancer; HR: Hazard ratio.

* Combined score based on peritumoral inflammation (1 point if score 0 or 1), Crohn's disease-like reaction (1 point if score 0 or 1 ) and global tumor stromal percentage ( 1 point if $>50 \%$ ) to give a maximum combined fibroinflammatory index score of 3 . Very few patients had a score of 3 , therefore this was combined with a score of 2 for statistical analysis.

**Adjusted for: sex (male/female), adjuvant chemotherapy use (yes/no), age $(<50,50-<60,60-<70,70-<80$, $\geq 80$ years), year of diagnosis (2004-2008), stage (II/III), family history of colorectal cancer (yes/no/unknown), MSI status (non MSI-high/MSI-high/unknown), extramural venous invasion (no/yes/unknown), ECOG status (0-1/2/3-4/unknown), differentiation (well-moderate/poor) and (for all-cause mortality analysis only) Charlson co-morbidity score (0-7). 
Figure 1.
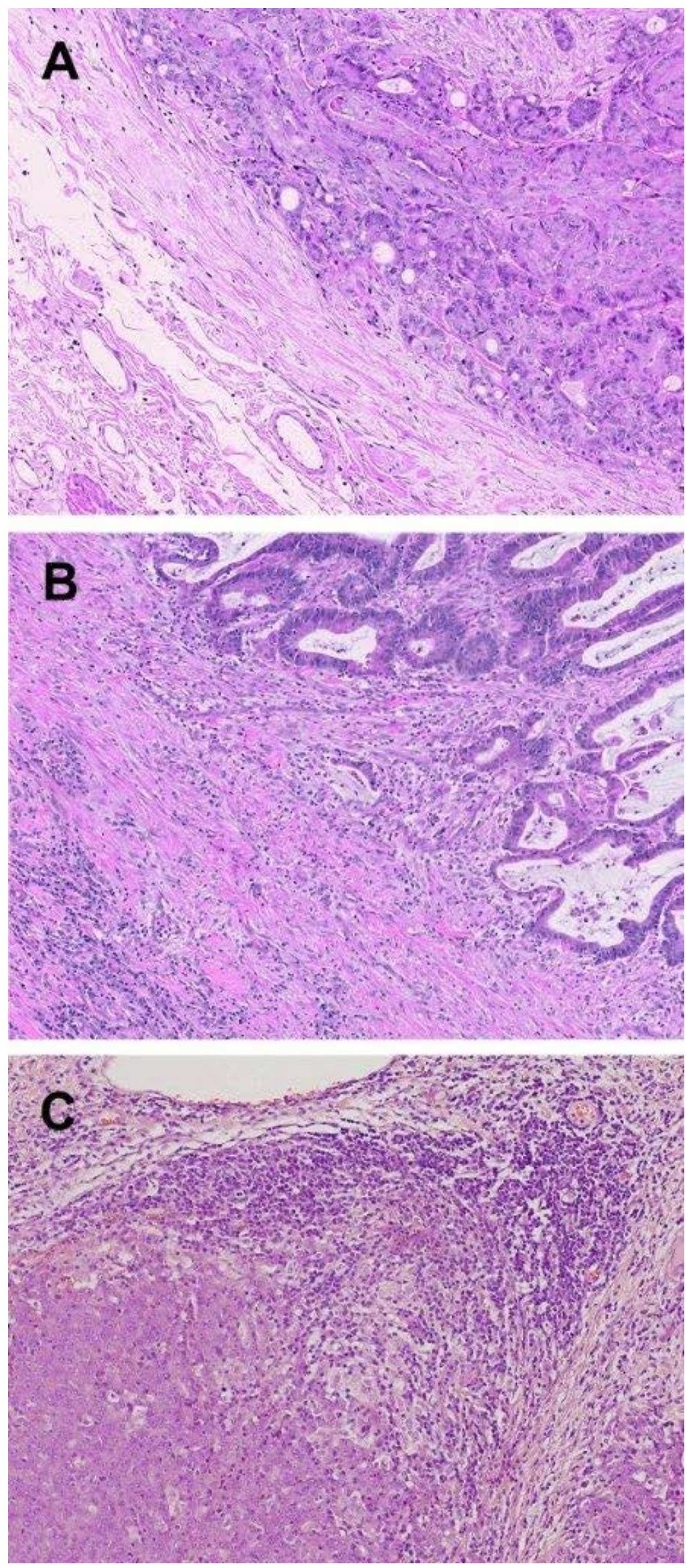
Figure 2.
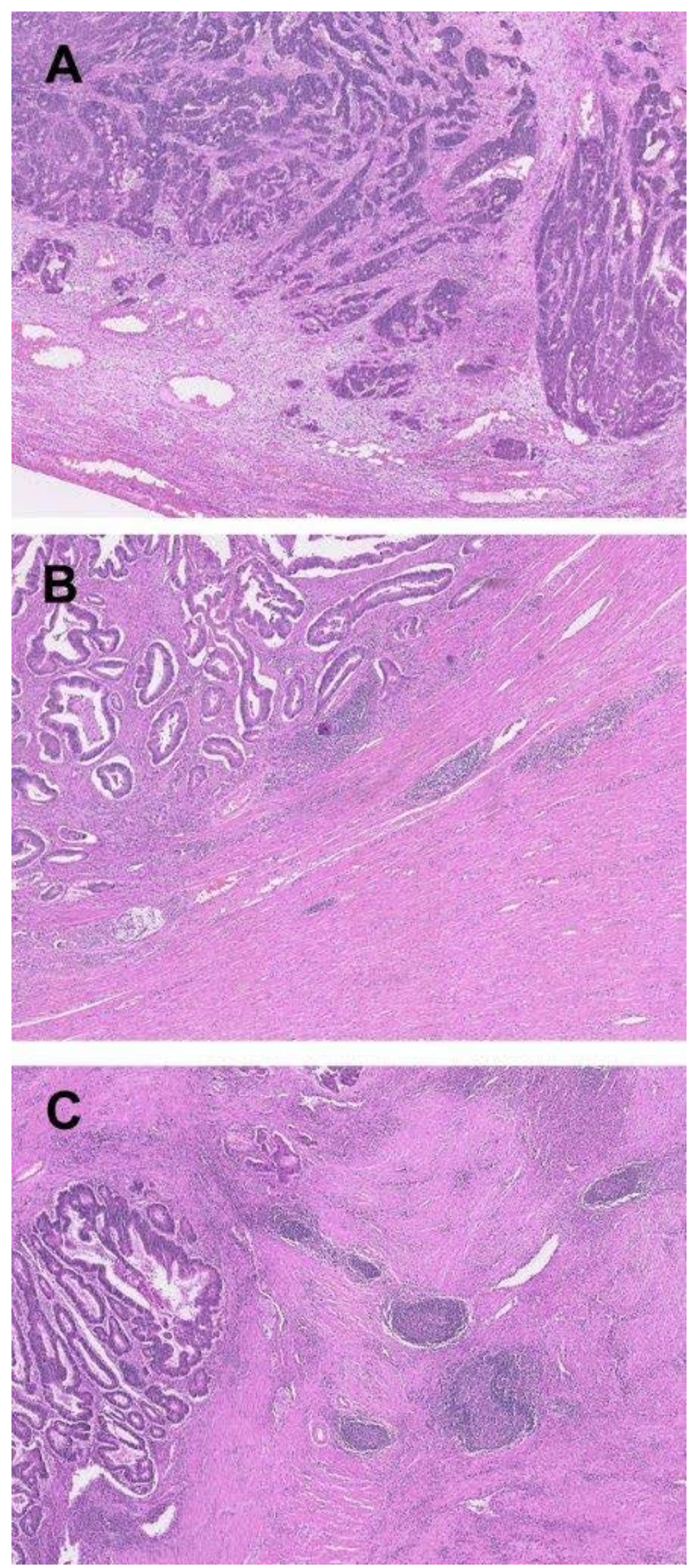
Figure 3.

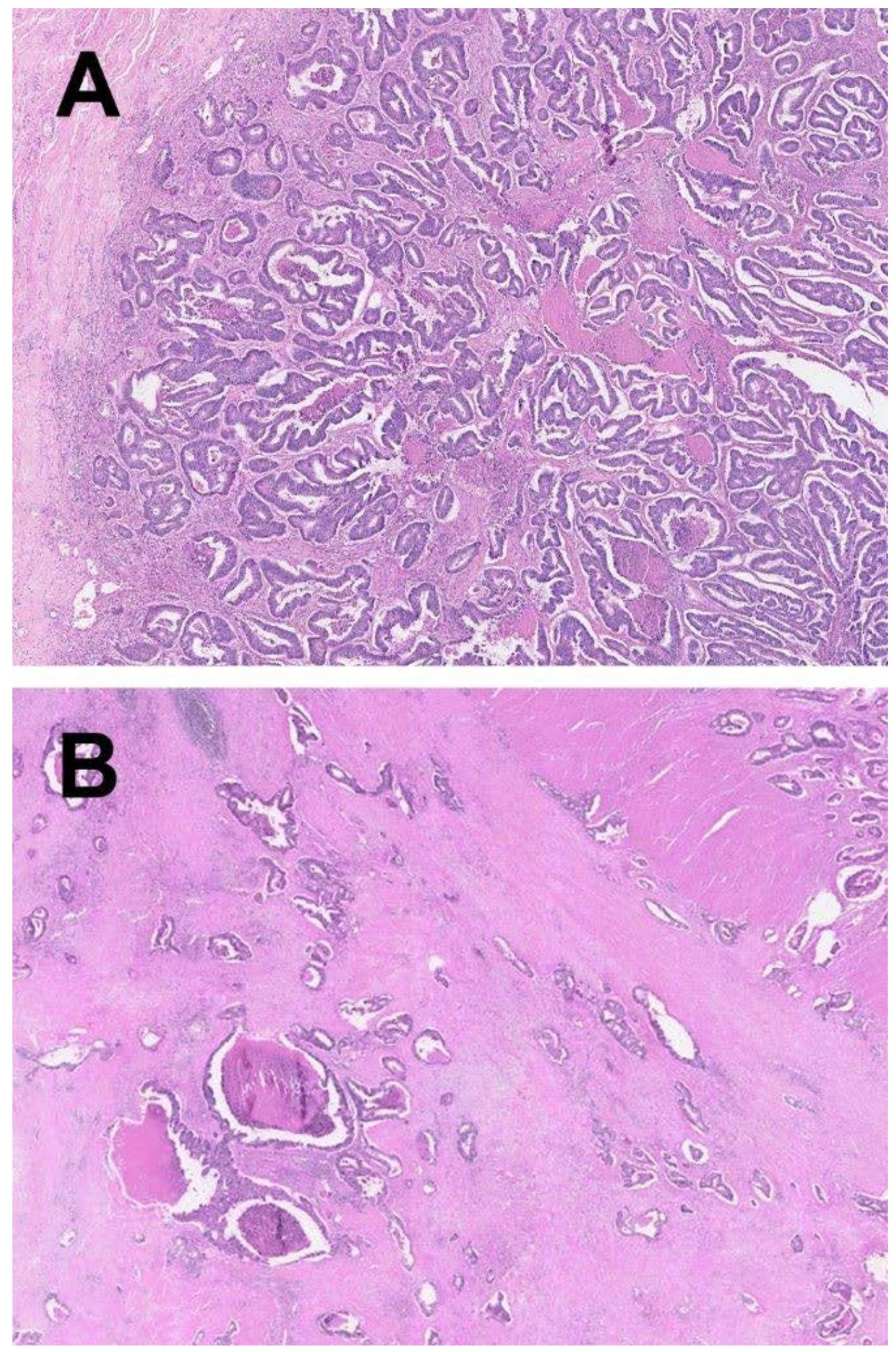


Figure 4. Kaplan Meier graph showing colorectal cancer-specific survival amongst Stage II and III colon cancer patients according to the combined fibro-inflammatory index score.

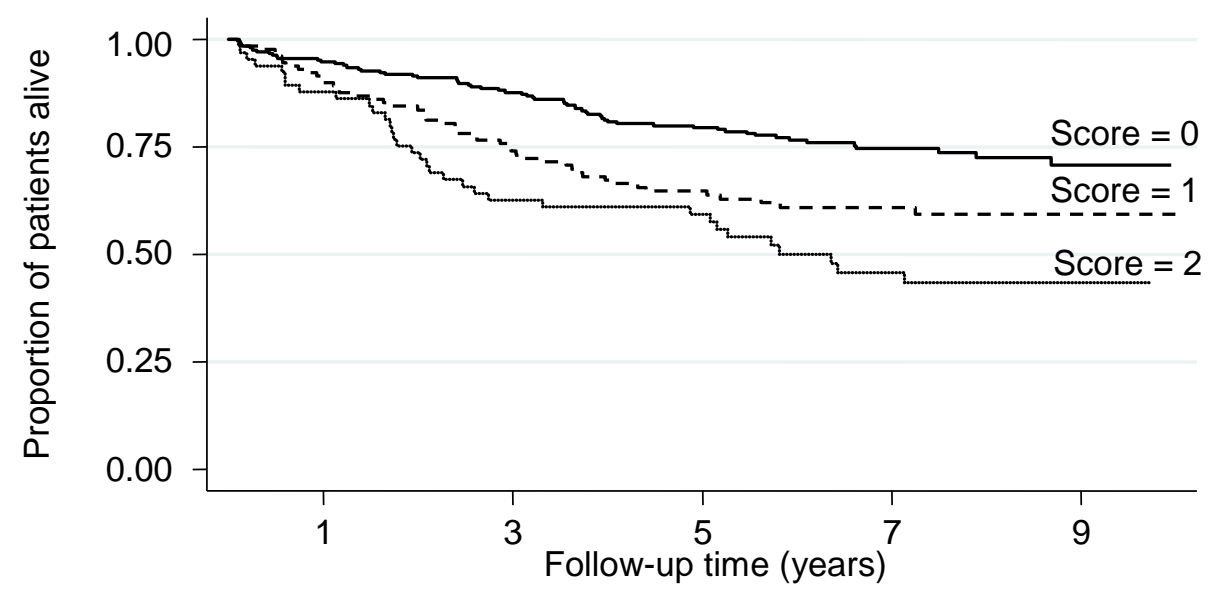

Number at risk Index score $=0$ Index score $=1$ Index score $=2$

56

209
89
39

179

86

29

39

34

40

20

10

1 
Figure 5. Risk of colorectal cancer-specific death according to morphological features in stage II and III colon cancer cases, stratified by microsatellite instability status.

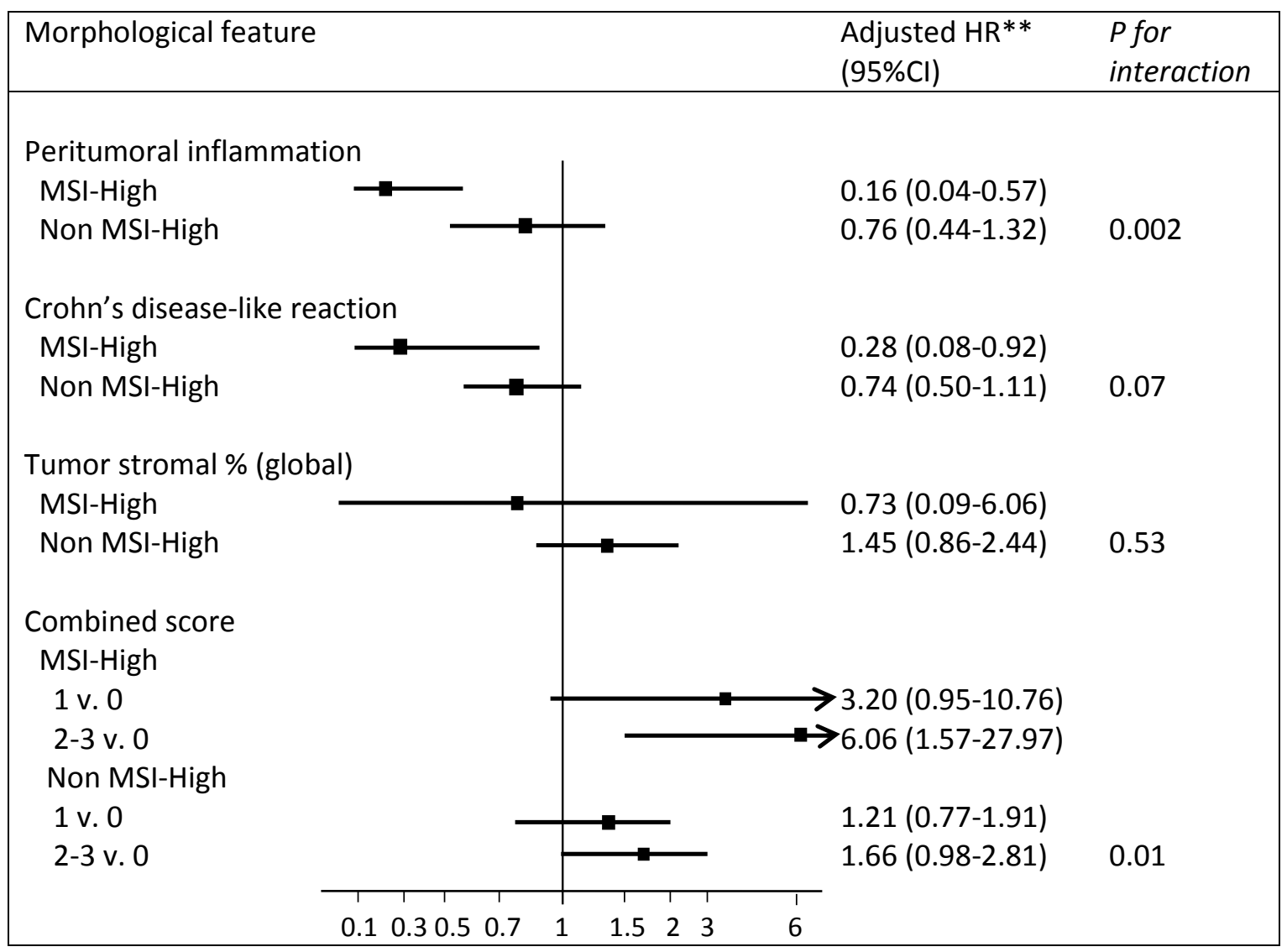

$\mathrm{Cl}$ : Confidence intervals; HR: Hazard ratio; MSI: Microsatellite instability. Results for peritumoral inflammation and Crohn's disease-like reaction are comparing scores $1 / 2 \mathrm{v} .0$ for each and results for tumor stromal percentage (global) are comparing $>50 \%$ v. $<50 \%$. Combined score is based on peritumoral inflammation ( 1 point if score 0 ), Crohn's disease-like reaction ( 1 point if score 0 ) and global tumor stromal percentage ( 1 point if $>50 \%$ ) to give a maximum combined fibroinflammatory index score of 3 . Very few patients had a score of 3, therefore this was combined with a score of 2 for statistical analysis.

**Adjusted for: sex (male/female), adjuvant chemotherapy use (yes/no - except for chemotherapy stratified analysis), age $(<50,50-<60,60-<70,70-<80, \geq 80$ years), year of diagnosis (2004-2008), stage (II/III - except for stage stratified analysis), family history of colorectal cancer (yes/no/unknown), MSI status (non MSI-high/MSI-high/unknown - except for MSI stratified analysis), extramural venous invasion (no/yes/unknown), ECOG status (0-1/2/3-4/unknown) and differentiation (well-moderate /poor). 
Supplementary Table 1. Risk of colorectal cancer-specific death according to microenvironmental features in stage II and III colon cancer cases, stratified by stage and adjuvant chemotherapy use.

\begin{tabular}{|c|c|c|c|c|c|}
\hline Morphological feature & $\begin{array}{l}\text { Number of } \\
\text { patients alive/ } \\
\text { CRC deaths }\end{array}$ & $\begin{array}{l}\text { Adjusted HR** } \\
(95 \% \mathrm{Cl})\end{array}$ & $\begin{array}{l}\text { Number of } \\
\text { patients alive/ } \\
\text { CRC deaths }\end{array}$ & $\begin{array}{l}\text { Adjusted HR** } \\
(95 \% \mathrm{Cl})\end{array}$ & $\begin{array}{l}\text { P for } \\
\text { interaction }\end{array}$ \\
\hline By stage & & Stage II & & Stage III & \\
\hline \multicolumn{6}{|c|}{ Peritumoral inflammation } \\
\hline Absent/Mild & $15 / 10$ & 1.00 & $7 / 17$ & 1.00 & \\
\hline Moderate/Severe & $145 / 55$ & $0.74(0.35-1.54)$ & $72 / 62$ & $0.41(0.22-0.76)$ & 0.10 \\
\hline \multicolumn{6}{|c|}{ Crohn's disease-like reaction } \\
\hline Absent/Mild & $42 / 25$ & 1.00 & $25 / 40$ & 1.00 & \\
\hline Moderate/Severe & $118 / 40$ & $0.75(0.44-1.28)$ & $54 / 39$ & $0.54(0.33-0.89)$ & 0.61 \\
\hline \multicolumn{6}{|c|}{ Tumor stromal \% (global) } \\
\hline$<50 \%$ & $147 / 52$ & 1.00 & $65 / 73$ & 1.00 & \\
\hline$>50 \%$ & $13 / 13$ & $1.74(0.90-3.40)$ & $14 / 18$ & $1.17(0.60-2.29)$ & 0.15 \\
\hline \multicolumn{6}{|l|}{ Combined score* } \\
\hline 0 & $107 / 30$ & 1.00 & $43 / 31$ & 1.00 & \\
\hline 1 & $38 / 23$ & $1.61(0.89-2.92)$ & $27 / 26$ & $1.55(0.86-2.75)$ & \\
\hline $2-3$ & $15 / 12$ & $1.81(0.88-3.75)$ & $9 / 22$ & $2.61(1.38-4.94)$ & 0.62 \\
\hline \multicolumn{2}{|c|}{ By adjuvant chemotherapy use } & No & & Yes & \\
\hline \multicolumn{6}{|c|}{ Peritumoral inflammation } \\
\hline Absent/Mild & $16 / 18$ & 1.00 & $6 / 9$ & 1.00 & \\
\hline Moderate/Severe & $141 / 87$ & $0.57(0.33-0.99)$ & $76 / 30$ & $0.34(0.14-0.81)$ & 0.58 \\
\hline \multicolumn{6}{|c|}{ Crohn's disease-like reaction } \\
\hline Absent/Mild & $42 / 47$ & 1.00 & $25 / 18$ & 1.00 & \\
\hline Moderate/Severe & $115 / 58$ & $0.62(0.41-0.94)$ & $57 / 21$ & $0.69(0.32-1.49)$ & 0.58 \\
\hline \multicolumn{6}{|c|}{ Tumor stromal \% (global) } \\
\hline$<50 \%$ & $144 / 86$ & 1.00 & $68 / 30$ & 1.00 & \\
\hline$>50 \%$ & $13 / 19$ & $1.52(0.87-2.66)$ & $14 / 9$ & $1.11(0.45-2.69)$ & 0.63 \\
\hline \multicolumn{6}{|l|}{ Combined score* } \\
\hline 0 & $103 / 48$ & 1.00 & $47 / 13$ & 1.00 & \\
\hline 1 & $39 / 32$ & $1.28(0.79-2.09)$ & $26 / 17$ & $2.01(0.83-4.84)$ & \\
\hline $2-3$ & $15 / 25$ & $2.23(1.32-3.77)$ & $9 / 9$ & $2.70(0.99-7.37)$ & 0.61 \\
\hline
\end{tabular}

Supplementary Table 1 legend.

$\mathrm{Cl}$ : Confidence intervals; CRC: Colorectal cancer; HR: Hazard ratio.

* Combined score based on peritumoral inflammation (1 point if score 0 or 1 ), Crohn's disease-like reaction ( 1 point if score 0 or 1 ) and global tumor stromal percentage ( 1 point if $>50 \%$ ) to give a maximum combined fibroinflammatory index score of 3 . Very few patients had a score of 3 , therefore this was combined with a score of 2 for statistical analysis.

**Adjusted for: Sex (male/female), adjuvant chemotherapy use (yes/no - except for chemotherapy stratified analysis), age ( $<50,50-<60,60-<70,70-<80, \geq 80$ years), year of diagnosis (2004-2008), stage (II/III - except for stage stratified analysis), family history of colorectal cancer (yes/no/unknown), MSI status (non MSI-high/MSI-high/unknown - except for MSI stratified analysis), extramural venous invasion (no/yes/unknown), ECOG status (0-1/2/3-4/unknown) and differentiation (moderate-well/poor). 